The Egyptian Journal of Hospital Medicine (January 2022) Vol. 86, Page 689-695

\title{
Does Ultrasound-Guided Continuous Suprascapular Nerve Block Affect Frozen Shoulder Rehabilitation Programme Outcome?
}

Hosam I. El Said Saber ${ }^{1 *}$, Amal Rashad ${ }^{1}$, Ebrahim Eltnany ${ }^{1}$, Reham M. Shaat ${ }^{2}$, Zaglool $\mathbf{A}^{\mathbf{1}}$

${ }^{1}$ Department of Anesthesia and Surgical Intensive Care, ${ }^{2}$ Department of Rheumatology and Rehabilitation, Faculty of Medicine, Mansoura University, Mansoura, Egypt.

*Corresponding author: Hosam I. El Said Saber, Mobile: +201005785775 - +201110383210, E-mail: hosamsaber@yahoo.com _hosamibrahim@mans.edu.eg,

\begin{abstract}
Background: Physical therapy (PT) is often recommended for patients with frozen shoulder. However, it could be painful for some patients, and this could hinder the rehabilitation programme. Some procedures like joint injection and suprascapular nerve block (SSNB) could alleviate pain during this setting. Objective: The purpose of this study was to compare the effectiveness of continuous SSNB plus PT compared to PT alone in managing frozen shoulder.

Patients and methods: A total of 76 patients with frozen shoulder were included in this study. They were divided into two groups: 38 patients in the injection group (IG) received SSNB via catheter before PT, while the remaining 38 participants in the control group (CG) received no block prior to PT (CG). The functional state of the shoulder joint was assessed via the constant shoulder scale before and just after PT, then one month later.

Results: General patient characteristics, including age, gender, BMI, comorbidities, and trauma history, were statistically comparable between the two groups. When we examined the constant scores of the two groups, we found that both had low scores before treatment, which increased immediately after treatment and then increased again one month later. Nonetheless, the injection group had a much greater increase than the control group.

Conclusion: When used with PT for the treatment of adhesive capsulitis, continuous SSNB is an effective option that enhances the response to PT. It is associated with better improvement in shoulder function.
\end{abstract}

Keywords: Frozen shoulder, Outcome, Suprascapular Nerve Block.

\section{INTRODUCTION}

In 1934, Codman invented the phrase "frozen shoulder" to characterize insidiously-onset painful shoulder diseases with shoulder movement restriction. The term adhesive capsulitis is now extensively used to describe the underlying pathology ${ }^{(1)}$.

Frozen shoulder affects women two to four times more than men and is more common in people aged 40 to 60 . It affects about $2 \%$ to $5 \%$ of the population ${ }^{(2)}$. The presence of progressive limited joint mobility and generalized pain despite conservative therapies is used to make the clinical diagnosis. A painful treatment process and a lengthy recovery, which can last up to two years, are characteristic features of this pathology ${ }^{(3)}$.

Frozen shoulder discomfort and spasm are routinely treated with physical therapy (PT) techniques such as analgesic currents that are used to apply superficial and deep heat. To enhance joint mobility and restore function, rehabilitation techniques such as stretching, strengthening exercises, and mobilization movements are also commenced ${ }^{(4)}$.

These procedures, on the other hand, are frequently associated with pain, which could hinder the patient from completing his planned rehabilitation programme. As a result, in the case of frozen shoulder, suprascapular nerve block (SSNB) or intraarticular injection therapy may be commenced for these patients prior to $\mathrm{PT}^{(5)}$.

About 70 percent of the shoulder area is innervated by the suprascapular nerve, which includes the acromioclavicular joint, the posterior and superior regions of the shoulder joint, and the joint capsule ${ }^{(6)}$. SSNB is an interventional procedure that reduces shoulder pain caused by various illnesses by blocking pain signal transmission from the shoulder joint to the corresponding segments in the spinal cord ${ }^{(7)}$. in $1941^{(8)}$.

This blockade technique was initially described

Although this block technique is not often used because of its complications (like injury to the supraclavicular nerve, pneumothorax, and vascular injuries), Dangoisse et al. modified the original technique, which led to a dramatic decrease in postprocedural complication rate $^{(9)}$. The local anesthetic injected during SSNB could be combined with steroids to prolong its action and enhance its efficacy ${ }^{(10)}$.

The goal of this study was to compare the efficacy of continuous SSNB plus PT versus PT alone in managing frozen shoulder patients.

This article is an open access article distributed under the terms and conditions of the Creative Commons Attribution (CC BY-SA) license (http://creativecommons.org/licenses/by/4.0/) 


\section{PATIENTS AND METHODS}

This study included a total of seventy-six patients diagnosed with adhesive capsulitis with shoulder pain and limited movement for at least one month. Patients were collected from the Pain Clinic or Outpatient Physical Therapy and Rehabilitation Clinics, Mansoura University. Patients with cervical radiculopathy, inflammatory joint disease, cardiac disorders, bleeding diathesis, or hypersensitivity to steroid and local anesthetics were excluded from this study. In addition, patients who reported intraarticular drug injections throughout the previous three months in the same tested shoulder joint were excluded as well.

During history taking, we focused on the presence of diabetes or previous history of trauma to the same shoulder, as the main two secondary causes of such condition.

\section{Ethical approval:}

The study was approved by the Institutional Review Board with Code Number (MD.18.05.49 July 2018), Faculty of Medicine, Mansoura University. The disease course, methods, management plan, and study benefits were all explained to the patients. A written informed consent was taken from each patient. This work has been carried out in accordance with the Code of Ethics of the World Medical Association (Declaration of Helsinki) for studies involving humans.

A PT program was scheduled for all patients, and it included hot packs application, ultrasound (US) for joint heating before mobilization techniques, transcutaneous electrical nerve stimulation (TENS), in addition to range of motion (ROM) and stretching exercises. A total of 15 sessions were performed for all participants (5 days weekly, for three consecutive weeks).

Using the closed envelopes, the patients were randomly assigned to one of two groups. The 38 patients in the injection group (IG) received SSNB via catheter prior to $\mathrm{PT}$, but the remaining 38 participants in the control group received no block prior to PT (CG).

In the IG group, a perineural 20-gauge catheter (B. Braun Melsungen AG, Melsungen, Germany) was placed in the scapular notch below the transverse scapular ligament (TSL) to create a continuous block of the SSN. Sonoscape A5 ultrasound equipment (Sonoscape, Guangdong, China) with a $1.8-6 \mathrm{MHz}$ transducer was used. There was no neuronal stimulation. The skin was cleansed with $70 \%$ alcohol, and the ultrasound (US) transducer was wrapped with a sterile bandage.

We placed the US probe on the scapula in the sitting posture to get the best possible transverse view of the suprascapular nerve (SSN) and suprascapular arteries. Following that, the depth of field (typically between $3-5 \mathrm{~cm}$ ) and focus range was optimized to enhance the machine imaging features.

The needle was inserted at the transducer's medial end and progressed at a shallow angle to the transducer from the medial insertion point to the inferiorlateral endpoint below the TSL in the scapular notch under real-time US guidance, allowing for a longer tunnel through the supraspinatus muscle. After a $5 \mathrm{ml}$ bolus of bupivacaine $2 \mathrm{mg} / \mathrm{ml}$ was administered through the needle, the catheter was pushed $1 \mathrm{~cm}$ beyond its tip. Under US supervision, the correct location of the catheter tip was confirmed when a $1 \mathrm{ml}$ bupivacaine 2 $\mathrm{mg} / \mathrm{ml}$ injectate induced expansion beneath the TSL after the needle was retracted.

At our outpatient clinic, the patient was examined every other day, and a recovery nurse assessed the dressing and catheter insertion site at the same time, instructing the patient to call the anesthesia department if anything changed. Bupivacaine was utilized as a local anesthetic, and triamcinolone was employed as a steroid adjuvant to extend the duration of the block. These two medicines were mixed into a single dosage (1 cc triamcinolone $+9 \mathrm{cc}$ bupivacaine). When necessary, similar doses were given before PT.

Physical therapy was provided by a licensed physical therapist with clinical experience. For 20 minutes, hot packing was applied over the shoulder area. TENS at a $100 \mathrm{~Hz}$-frequency, $15 \mathrm{~mA}$ amplitude, and 100 m.secs was administered to the anterior and posterior sides of the shoulder joint for a 20-minute duration. The patients were subjected to continuous ultrasound (via Intelect Mobile Combo machine) with a $5 \mathrm{~cm}$ transducer head that ran at a frequency of $1 \mathrm{MHz}$ and an intensity of $1.5 \mathrm{~W} / \mathrm{cm}$. The affected shoulder was subjected to slow circular movements with a transmission gel for 10 minutes.

In the supine position, pendulum exercises, passive ROM activities, and passive stretching exercises for the abductor, and external, and internal rotator muscle groups were started until pain tolerance was reached. In the second week, active ROM and stretching activities were included in the treatment. Isometric strengthening exercises targeting the same muscle groups were combined with stretching activities in the third week. Later in the day, and using the help of the other healthy arm, the same exercises were repeated by all subjects. After treatment, the patients were instructed to perform the recommended programme twice daily at home.

A researcher blinded to the patient groups assessed all patients for functional status before, immediately after PT, and one month afterward, for a total of three times. The Constant Shoulder Scale ${ }^{(11)}$, 
which was established to evaluate the functional recovery of the shoulder, ROM, and its functional condition, was used in our study. It is graded on a scale of 0 to 100 , with higher scores signifying better results. The four components of the score are pain, daily activities, range of motion, and strength.

\section{Statistical analysis}

The Statistical Package for the Social Sciences (SPSS, IBM, Inc, Chicago, USA) version 26 for windows was used for data collecting, tubulation, and analysis. The Kolmogorov-Smirnov test was used to check for normalcy in quantitative data, which was then reported as mean and standard deviation (SD).
Percentage and frequency were used to express categorical data. For comparison between the 2 groups, independent $\mathrm{t}$ - test was utilized. When comparing categorical data, the Chi square test was utilized. For comparing quantitative data at 3 time points, the repeated measures ANOVA test was used. It was determined that probability $(\mathrm{P}<0.05)$ was statistically significant.

\section{RESULTS}

There was no statistically significant difference between the two groups as regard age, anthropometric measures, gender, occupation, dominant hand, sick side, diabetic state, or history of trauma (Tables $1-4)$.

Table (1): Patient characteristics between the two study groups

\begin{tabular}{|c|c|c|c|c|}
\hline & & \multicolumn{2}{|c|}{ Groups } & \multirow{2}{*}{ P-value } \\
\hline & & IG $(\mathrm{N}=38)$ & $C G(N=38)$ & \\
\hline \multicolumn{2}{|l|}{ Age (years) } & $52.58 \pm 6.78$ & $51.37 \pm 5.80$ & 0.406 \\
\hline \multirow{2}{*}{ Gender } & Male & $28.9 \%$ & $13.2 \%$ & \multirow{2}{*}{0.091} \\
\hline & Female & $71.1 \%$ & $86.8 \%$ & \\
\hline \multirow{3}{*}{ Residence } & Housewife & $42.1 \%$ & $52.6 \%$ & \multirow{3}{*}{0.132} \\
\hline & Retired & $15.8 \%$ & $2.6 \%$ & \\
\hline & working & $42.1 \%$ & $44.7 \%$ & \\
\hline \multicolumn{2}{|l|}{ Weight (Kg) } & $81.84 \pm 6.09$ & $81.97 \pm 3.95$ & 0.911 \\
\hline \multicolumn{2}{|l|}{ Height (cm) } & $158.32 \pm 3.27$ & $157.45 \pm 2.58$ & 0.202 \\
\hline \multicolumn{2}{|l|}{ BMI $\left(\mathrm{kg} / \mathrm{m}^{2}\right)$} & $32.83 \pm 1.57$ & $33.16 \pm 1.24$ & 0.313 \\
\hline
\end{tabular}

Quantitative data are expressed as mean \pm standard deviation and qualitative data are expressed as frequency and percentage.

No statistically significant difference was also found between the 2 groups as regard diabetes mellitus and history of trauma (Table 2).

Table (2): Risk factors for frozen shoulder in the two study groups

\begin{tabular}{|c|c|c|c|c|c|c|}
\hline & & & & & & D voluo \\
\hline & & & $=38)$ & & $=38)$ & \\
\hline DM & No & 15 & $39.5 \%$ & 14 & $36.8 \%$ & 0813 \\
\hline Divi & Yes & 23 & $60.5 \%$ & 24 & $63.2 \%$ & \\
\hline Troum & $\mathrm{No}$ & 15 & $39.5 \%$ & 15 & $39.5 \%$ & 1 \\
\hline Trauma & Yes & 23 & $60.5 \%$ & 23 & $60.5 \%$ & 1 \\
\hline
\end{tabular}

DM: Diabetes mellitus

Table (3): Affected and dominant hand in the two study groups

Groups

IG CG

$(\mathbf{N}=38)$

$(\mathbf{N}=38)$

\begin{tabular}{lccccc}
\hline \multirow{2}{*}{$\begin{array}{l}\text { Dominant } \\
\text { hand }\end{array}$} & Right & 36 & $94.7 \%$ & 37 & $97.4 \%$ \\
\cline { 2 - 6 } Diseased side & Left & 2 & $5.3 \%$ & 1 & $2.6 \%$ \\
\cline { 2 - 6 } & Right & 33 & $86.8 \%$ & 35 & $92.1 \%$ \\
\hline
\end{tabular}


Table 4 shows that the disease duration was statistically comparable between the two groups.

Table (4): Duration of disease between the study groups

\begin{tabular}{|c|c|c|c|c|c|c|}
\hline & & \multicolumn{4}{|c|}{ Groups } & \multirow[b]{2}{*}{ P-value } \\
\hline & & \multicolumn{2}{|c|}{$\begin{array}{c}\text { IG } \\
(\mathrm{N}=38)\end{array}$} & \multicolumn{2}{|c|}{$\begin{array}{c}\mathbf{C G} \\
(\mathrm{N}=38)\end{array}$} & \\
\hline \multirow{3}{*}{ Duration } & 1-3 months & 21 & $55.3 \%$ & 13 & $34.2 \%$ & \multirow{3}{*}{0.179} \\
\hline & 3-6 months & 8 & $21.1 \%$ & 11 & $28.9 \%$ & \\
\hline & $\begin{array}{l}\text { more than six } \\
\text { months }\end{array}$ & 9 & $23.7 \%$ & 14 & $36.8 \%$ & \\
\hline
\end{tabular}

When we examined the two groups' constant scores, we discovered that both had low scores before therapy. In both groups, the score improved immediately after therapy and improved again one month later. Nonetheless, the injection group had a greater rate than the control group. That is to say; the injection group showed a greater improvement than the control group (table 5).

Table (5): Constant score evaluation between the two study groups

\begin{tabular}{lccc}
\hline & \multicolumn{2}{c}{ Groups } & \multirow{2}{*}{ P-value } \\
\cline { 2 - 3 } & $\begin{array}{c}\text { IG } \\
(\mathbf{N}=\mathbf{3 8})\end{array}$ & $\begin{array}{c}\text { CG } \\
(\mathbf{N}=\mathbf{3 8})\end{array}$ & \multirow{2}{*}{0.281} \\
\hline Pre-treatment & $48.39 \pm 3.63$ & $47.53 \pm 3.27$ & $0.004^{*}$ \\
\hline Immediately after treatment & $76.47 \pm 5.98$ & $72.26 \pm 6.29$ & $<0.001^{*}$ \\
\hline One month after treatment & $91.61 \pm 3.05$ & $80.97 \pm 5.42$ & \\
\hline Repeated-measures & $\mathrm{P}<0.001$ & $\mathrm{P}<0.001$ & \\
\hline ANOVA & $\mathrm{p} 1<0.001$ & $\mathrm{p} 1<0.001$ & $\mathrm{p} 2<0.001$ \\
Interclass correlation & $\mathrm{p} 2<0.001$ & $\mathrm{p} 3<0.001$ & $\mathrm{p} 3<0.001$
\end{tabular}

P1: Comparison between the constant score before treatment and the score immediately following therapy.

P2: Comparison between the constant score before treatment and the constant score one month later.

P3: Comparison between the constant score immediately after treatment and the score one month later. 


\section{DISCUSSION}

Frozen shoulder is a painful condition marked by glenohumeral joint synovitis and shoulder capsule thickening, as well as a complete or near-complete absence of motion. In randomized trials, no medication has been shown to be successful in terms of curing or shortening disease course ${ }^{(5,12)}$. However, a series of patients have reported positive outcomes following open or arthroscopic capsulotomy, manipulation under anesthetic, and prompt, rigorous physiotherapy ${ }^{(13,14)}$.

Pain relief sufficient enough to allow shoulder PT is difficult to acquire with oral medicine or patientcontrolled analgesia. Other researches have reported using a cervical epidural catheter, a subacromial catheter with lidocaine infusion, and an interscalene catheter to provide analgesia. However, respiratory depression and hypotension are possible dangers and adverse effects of epidural and interscalene infusions, and both procedures result in a significant loss in arm motor performance ${ }^{(\mathbf{1 3},}$ 15).

From an anesthetic standpoint, three methods of regional block techniques exist to achieve adequate pain management for painful shoulder conditions. The first option would be the perineural catheter, which could be inserted at the interscalene level, where the brachial plexus is located. However, it has multiple cons including, the risk of diaphragmatic paralysis due to phrenic nerve blockade; the motor block which hinders active rehabilitation exercises; prolonged Horner's syndrome; and catheter-related complications like displacement ${ }^{(16)}$.

Another alternative during the recuperation period is to implant a cervical epidural catheter, which would deliver a continuous infusion of local anesthetic agents or opioid drugs. In a retrospective study, this was determined to be a safe and effective treatment for postoperative pain control and rehabilitation (15). However, most hospitals would not consider providing this method to outpatients to be safe, and we do not currently have the logistical or organizational infrastructure in place to treat outpatients with such a technique.

The third viable option is to block the SSN's sensory segment, which serves the scapula and acromioclavicular joint and provides about $70 \%$ of the nerve supply for the posterior and superior sections of the shoulder (single-shot block or catheter implantation) (17). The subacromial bursa, coracohumeral ligament, and posterior side of the acromioclavicular joint capsule are supplied by the superior articular branch of the SSN, whereas the inferior branch of the same nerve provides supply for the posterior joint capsule. The supra- and infraspinatus muscles are likewise innervated by the $\mathrm{SSN}$; however, the inferior and anterior shoulder areas are not ${ }^{(18)}$. The results of this trial demonstrated that physical therapy combined with exercises was effective in lowering pain and improving functional status, with SSNB before PT increasing efficacy even further. The findings of our study are similar to those of most previous studies regarding the beneficial impact of PT on patients with adhesive capsulitis ${ }^{(\mathbf{1 9 - 2 1 )}}$.

A variety of modalities have been used in conjunction with PT to reduce or eliminate pain, enhance range of motion in the shoulders, and improve functional status ${ }^{(19)}$. TENS, hot packs, and ultrasound are routinely utilized and have been demonstrated to be successful therapies in adhesive capsulitis, despite the lack of clear consensus in the research. The former is used for pain relief, while the two other methods are used for superficial and deep tissue heating, respectively ${ }^{(21,22)}$. Moreover, stretching and range of motion exercises to prevent additional loss of range of motion, as well as strengthening exercises to counter weakness in the muscles of the shoulder girdle, are usually advised $(20,23,24)$

In agreement with the previous studies, the PT in the current study consisted of TENS, hot packs, US, ROM exercises and stretching.

Hsieh and his colleagues evaluated the effect of adding hyaluronic acid injection to PT in cases diagnosed with adhesive capsulitis. Hyaluronate injections were commenced prior to PT therapies, which included electrical therapy, heat, and exercises. Adding hyaluronic acid was not associated with significant improvements compared to cases receiving PT only ${ }^{(21)}$.

Nonetheless, in the current investigation, it was discovered that using SSNB prior to PT boosted the treatment's results, suggesting that pain alleviation provided by nerve blockade may be an essential element in the treatment's response. The current findings contradict those of Carette et al. who found that 12 physiotherapy sessions had no significant benefit in cases with adhesive capsulitis over a one-year follow-up duration compared to placebo. Nonetheless, the authors stated that a combination of steroid injection and physical therapy improved shoulder ROM more quickly (25).

SSNB is currently recommended for various painful shoulder joint conditions, including rotator cuff lesion (10), adhesive capsulitis (26), and hemiplegic shoulder (27). It is a therapeutic option that is safe, effective, and could be repeated at a low cost. A previous study discovered that SSNB is effective in pain reduction and functional improvement faster than intraarticular corticosteroid injections in cases with adhesive capsulitis (26).

Taskaynatan and his associates included 60 cases with non-specific shoulder pain. They compared the effect of SSNB versus intraarticular steroid injection and found that both groups expressed significant 
improvements in joint function, ROM, and pain after one month ${ }^{(28)}$. Nearly all of the indicators related to pain and functional status improved more in the block group than in the controls in our trial, confirming the efficacy of SSNB as seen in the previous investigations.

Likewise, pain scores dropped considerably in the SSNB group compared to controls in the study of Dahan $\boldsymbol{e t}$ al. which included 34 individuals with adhesive capsulitis. The difference in functional capacity was not substantial, and there was no change in shoulder ROM. Apart from brief vagal stimulating effects and local sensitivity at the injection site, no adverse effects were noted ${ }^{(29)}$.

No side effects or complications were reported by the injection patients in this trial, indicating that SSNB is a safe therapeutic option. The Constant Shoulder Scale also showed a significant increase in both study groups.

When the groups were compared in the current study, the IG group received significantly higher scores. According to the findings of Dahan and his associates, pain reduction is the primary benefit of SSNB, which aids in the maintenance of pain-free PT interventions ${ }^{(29)}$.

In a previous study that included 83 cases with adhesive capsulitis, bupivacaine was used for SSNB in conjunction with steroids in one group and then compared to the other group that received a placebo. Joint movement, function, and pain showed significant improvement in the intervention group, and that was evident at one-, four-, and twelve-week follow-up visits. The nerve blockade showed more durable effects when compared to pharmacological therapy. This could be explained by diminished central sensitivity in the dorsal horn neurons or desensitization caused by lower peripheral pain signals ${ }^{(30)}$.

In the current study, long-acting bupivacaine and steroid were used. According to certain research, combining corticosteroids with local anesthetics increases the efficiency of nerve blockade by 1.5 to 2 times and reduces pain scores ${ }^{(\mathbf{3 1}, 32)}$. This effect could be linked to both local and systemic decrease of neural transmission at nonmyelinated nociceptive $\mathrm{C}$ fibers by local steroid application, as several experimental studies have shown ${ }^{(33,34)}$. As a result, in this investigation, the combination with steroids was recommended to prolong and enhance the analgesic actions of local anesthetic injected during nerve block.

The small sample size and the short follow-up period of this study may restrict its findings. Larger randomized trials are needed to cover these drawbacks. Future large-scale studies will be needed to address the question of perineural catheter patency over time, unintentional discontinuation rates, and potential side effects.

\section{CONCLUSION}

The current study's findings suggest that when combined with PT, continuous SSNB is a safe and welltolerated technique for improving pain and functional status in individuals with adhesive capsulitis.

\section{Conflict of interest}

The authors of this study have reported no conflict of interest.

\section{REFERENCES}

1. Le H, Lee S, Nazarian A, Rodriguez E (2017): Adhesive capsulitis of the shoulder: review of pathophysiology and current clinical treatments. Shoulder Elbow, 9(2):75-84.

2. Zreik N, Malik R, Charalambous C (2016): Adhesive capsulitis of the shoulder and diabetes: a meta-analysis of prevalence. Muscles Ligaments Tendons J., 6(1):26-34.

3. Ramirez J (2019): Adhesive capsulitis: diagnosis and management. Am Fam Physician, 99(5):297300.

4. Chan H, Pua P, How C ( 2017): Physical therapy in the management of frozen shoulder. Singapore Med J., 58(12):685-9.

5. Challoumas D, Biddle M, McLean M, Millar N (2020): Comparison of treatments for frozen shoulder: A systematic review and meta-analysis. JAMA Netw Open, 3(12):e2029581.

6. Tran J, Peng P, Agur A (2019): Anatomical study of the innervation of glenohumeral and acromioclavicular joint capsules: implications for image-guided intervention. doi: 10.1136/rapm2018-100152.

7. Date A, Rahman L (2020): Frozen shoulder: overview of clinical presentation and review of the current evidence base for management strategies. Future Sci OA., 6(10):Fso647.

8. Chan C, Peng P (2011): Suprascapular nerve block: a narrative review. Reg Anesth Pain Med., 36(4):358-73.

9. Dangoisse M, Wilson D, Glynn C (1994): MRI and clinical study of an easy and safe technique of suprascapular nerve blockade. Acta Anaesthesiol Belg., 45(2):49-54.

10. Fernandes M, Barbosa M, Sousa A, Ramos G (2012): Suprascapular nerve block: important procedure in clinical practice. Rev Bras Anestesiol., 62(1):96-104.

11. Kirkley A, Griffin S, Dainty K (2003): Scoring systems for the functional assessment of the shoulder. Arthroscopy, 19(10):1109-20.

12. Robinson $C$, Seah $K$, Chee $Y$, Hindle $P$, Murray I (2012): Frozen shoulder. J Bone Joint Surg Br., 94(1):1-9. 
13. Andersen N, Søjbjerg J, Johannsen H, Sneppen O (1998): Frozen shoulder: arthroscopy and manipulation under general anesthesia and early passive motion. J Shoulder Elbow Surg., 7(3):21822.

14. Baums M, Spahn G, Nozaki M, Steckel H, Schultz W, Klinger $\mathbf{H}$ (2007): Functional outcome and general health status in patients after arthroscopic release in adhesive capsulitis. Knee Surg Sports Traumatol Arthrosc., 15(5):638-44.

15. Narouze S, Govil H, Guirguis $M$, Mekhail $N$ (2009): Continuous cervical epidural analgesia for rehabilitation after shoulder surgery: a retrospective evaluation. Pain Physician,12(1):189-94.

16. Tuominen M, Haasio J, Hekali R, Rosenberg $P$ (1989): Continuous interscalene brachial plexus block: clinical efficacy, technical problems and bupivacaine plasma concentrations. Acta Anaesthesiol Scand., 33(1):84-8.

17. Gorthi V, Moon Y, Kang J (2010): The effectiveness of ultrasonography-guided suprascapular nerve block for perishoulder pain. Doi:10.3928/01477447-20100225-11.

18. Børglum J, Bartholdy A, Hautopp H, Krogsgaard M, Jensen K (2011): Ultrasound-guided continuous suprascapular nerve block for adhesive capsulitis: one case and a short topical review. Acta Anaesthesiol Scand., 55(2):242-7.

19. Pajareya K, Chadchavalpanichaya N, Painmanakit S, Kaidwan C, Puttaruksa P, Wongsaranuchit $Y$ (2004): Effectiveness of physical therapy for patients with adhesive capsulitis: a randomized controlled trial. J Med Assoc Thai., 87(5):473-80.

20. Dundar U, Toktas H, Cakir T, Evcik D, Kavuncu V (2009): Continuous passive motion provides good pain control in patients with adhesive capsulitis. Int J Rehabil Res., 32(3):193-8.

21. Hsieh L, Hsu W, Lin Y, Chang H, Chen C, Huang $V$ (2012): Addition of intra-articular hyaluronate injection to physical therapy program produces no extra benefits in patients with adhesive capsulitis of the shoulder: a randomized controlled trial. Arch Phys Med Rehabil., 93(6):957-64.

22. Hsu J, Anakwenze O, Warrender W, Abboud J (2011): Current review of adhesive capsulitis. J Shoulder Elbow Surg., 20(3):502-14.

23. Russell S, Jariwala A, Conlon R, Selfe J, Richards J, Walton M (2014): A blinded, randomized, controlled trial assessing conservative management strategies for frozen shoulder. J Shoulder Elbow Surg., 23(4):500-7.
24. Struyf F, Meeus M (2014): Current evidence on physical therapy in patients with adhesive capsulitis: what are we missing? Clin Rheumatol., 33(5):593600.

25. Carette S, Moffet $\mathbf{H}$, Tardif J, Bessette L, Morin F, Frémont P, et al. (2003): Intraarticular corticosteroids, supervised physiotherapy, or a combination of the two in the treatment of adhesive capsulitis of the shoulder: a placebo-controlled trial. Arthritis Rheum., 48(3):829-38.

26. Jones D, Chattopadhyay C (1999): Suprascapular nerve block for the treatment of frozen shoulder in primary care: a randomized trial. Br J Gen Pract., 49(438):39-41.

27. Adey-Wakeling Z, Crotty M, Shanahan E (2013): Suprascapular nerve block for shoulder pain in the first year after stroke: a randomized controlled trial. Stroke, 44(11):3136-41.

28. Taskaynatan M, Yilmaz B, Ozgul A, Yazicioglu K, Kalyon T (2005): Suprascapular nerve block versus steroid injection for non-specific shoulder pain. Tohoku J Exp Med.,205(1):19-25.

29. Dahan T, Fortin L, Pelletier $M$, Petit $M$, Vadeboncoeur R, Suissa S (2000): Double blind randomized clinical trial examining the efficacy of bupivacaine suprascapular nerve blocks in frozen shoulder. J Rheumatol., 27(6):1464-9.

30. Shanahan EM, Ahern M, Smith M, Wetherall M, Bresnihan B, FitzGerald O (2003): Suprascapular nerve block (using bupivacaine and methylprednisolone acetate) in chronic shoulder pain. Ann Rheum Dis., 62(5):400-6.

31. Parrington S, O'Donnell D, Chan V, BrownShreves D, Subramanyam R, Qu M, et al. (2010): Dexamethasone added to mepivacaine prolongs the duration of analgesia after supraclavicular brachial plexus blockade. Reg Anesth Pain Med., 35(5):4226.

32. Vieira P, Pulai I, Tsao G, Manikantan P, Keller B, Connelly N (2010): Dexamethasone with bupivacaine increases duration of analgesia in ultrasound-guided interscalene brachial plexus blockade. European journal of anaesthesiology, 27(3):285-8.

33. Johansson A, Hao J, Sjölund B (1990): Local corticosteroid application blocks transmission in normal nociceptive C-fibres. Acta Anaesthesiol Scand., 34(5):335-8.

34. Movafegh A, Razazian M, Hajimaohamadi F, Meysamie A (2006): Dexamethasone added to lidocaine prolongs axillary brachial plexus blockade. Anesth Analg., 102(1):263-7. 